\title{
An Efficient Characterization of Fuzzy Temporal Interval Relations
}

\author{
Steven Schockaert, Martine De Cock, and Etienne E. Kerre
}

\begin{abstract}
Fuzzy temporal interval relations have been defined to support temporal knowledge representation and reasoning in the presence of vagueness. The most important impediment to use these fuzzy relations in real-world applications is the lack of a characterization that is both easy to implement and computationally efficient. In this paper, we provide such a characterization for the important class of piecewise linear fuzzy time intervals, which covers all types of fuzzy time intervals that we are likely to encounter in applications. Furthermore, we discuss a more elegant characterization for the special case of trapezoidally shaped fuzzy intervals.
\end{abstract}

\section{INTRODUCTION}

Very often the temporal information that is at our disposal is of a qualitative nature. A typical example is temporal information that is extracted from natural language (e.g. [1]). Allen [2] has proposed a set of 13 jointly exhaustive and mutual exclusive qualitative relations between intervals of the real line to reason with this kind of information. For example, if we know that $A$ happened before $B$, and $C$ happened during $B$, we may deduce that $A$ happened before $C$. The underlying assumption is that the time span of events can be modelled as an interval. In practice however, this is not always true, as temporal information is sometimes pervaded with uncertainty and vagueness.

In [3], Dubois and Prade discuss how Allen's definitions of the qualitative interval relations can be generalized when the endpoints of the intervals are uncertain. For example, assume that we know that event $A$ lasted from just before 10.15 am to a little over $1.00 \mathrm{pm}$, and $B$ lasted from around 10.00 am till the early evening. In the approach taken in [3], the endpoints of the time spans of $A$ and $B$ are represented as possibility distributions, which allows to express for example the possibility that event $A$ happened during $B$. In [4] Badaloni and Giacomin propose a framework for qualitative reasoning with uncertain temporal relations, which allows to deduce, for example, the possibility that $A$ happened before $C$ when we only know that the possibility that $A$ happened before $B$ is 0.6 and the possibility that $B$ happened during $C$ is 0.8 .

On the other hand, temporal information can also be affected by vagueness, in at least two different ways. First of all, the qualitative relations may be vague, expressing for example that $A$ and $B$ began at approximately the same time, or that $A$ happened long before $B$. As shown in [5], this can be modelled by making use of a graded inequality relation. Secondly, the time span of events may be vague. For example historical events tend to have a gradual beginning

\footnotetext{
The authors are with the Department of Applied Mathematics and Computer Science, Ghent University, Krijgslaan 281 (S9), B-9000 Gent, Belgium (email: Steven.Schockaert,Martine.DeCock,Etienne.Kerre@UGent.be).
}

and/or ending (e.g. the Cold War, the Great Depression, the Russian Revolution, ...). In [6] it was suggested to represent the time span of such vague events as fuzzy sets, which we call fuzzy intervals in this context, and generalized definitions of the qualitative interval relations were given. Alternative definitions of the interval relations which cope to some extent with vague temporal relations as well, were given in [7]. As pointed out in [5], approaches for ranking fuzzy numbers may also be suitable for this purpose (e.g. [8]). It seems however that in all of these approaches, important properties of the original interval relations are lost, which makes them unsuitable as a basis for qualitative reasoning. Therefore in [9], we have proposed alternative definitions, based on the notion of relatedness measures for fuzzy sets [10].

One of the most important limitations of these definitions for practical applications is that the fuzzy temporal relations are computationally rather expensive to evaluate. In this paper we introduce a computationally efficient characterization of these fuzzy temporal relations for an important class of fuzzy intervals. In Section II, we first recall the definitions of the fuzzy temporal interval relations that were introduced in [9], and generalized in [11]. Next, in Section III we provide a characterization of these fuzzy temporal interval relations for arbitrary piecewise linear fuzzy intervals. This gives us a method to process fuzzy temporal information in an efficient way, as in real-world applications the time spans of vague events will likely be represented by piecewise linear fuzzy intervals. Moreover, every fuzzy interval can be approximated by a piecewise linear fuzzy interval with an arbitrary high precision. In Section IV we discuss a special class of piecewise linear fuzzy intervals, namely trapezoidally shaped fuzzy intervals. Although the resulting characterization is less general, it is more elegant, and thus more apt to be used in theoretical arguments. Finally, Section $\mathrm{V}$ concludes the paper.

\section{FUZZY TEMPORAL RELATIONS}

\section{A. Crisp time intervals}

Qualitative relations between time intervals are usually defined as constraints on the boundary points of these intervals [2]. For example, we say that $A=\left[a^{-}, a^{+}\right]$is before $B=\left[b^{-}, b^{+}\right]$iff $a^{+}<b^{-}$, and that $A$ happened during $B$ iff $b^{-}<a^{-}$and $a^{+}<b^{+}$. Vague temporal relations like $A$ happened long before $B$ can be represented by using a fuzzy relation that expresses the degree to which one time point is long before another [5]. In the following, we use the fuzzy 


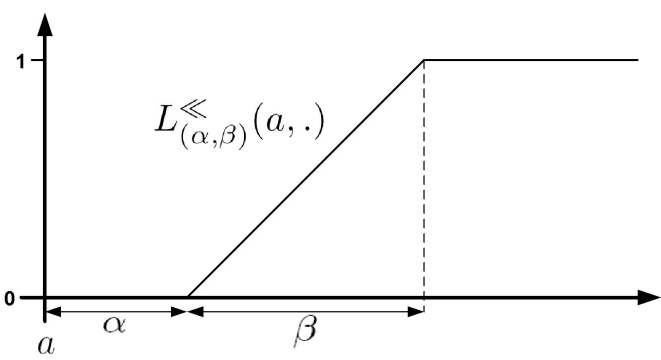

(a) $L_{(\alpha, \beta)}^{\ll}(a,$.

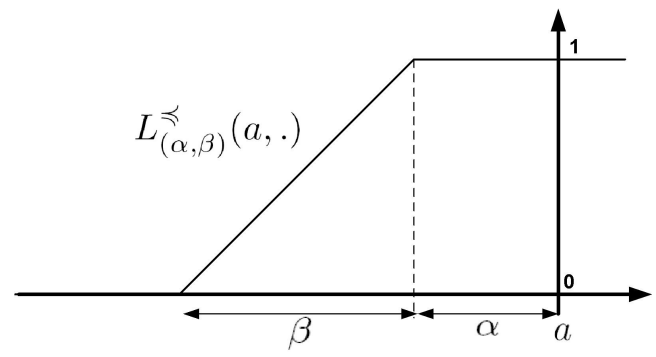

(b) $L_{(\alpha, \beta)}^{\preccurlyeq}(a,$.

Fig. 1. The fuzzy relations $L_{(\alpha, \beta)}^{\ll}$ and $L_{(\alpha, \beta)}^{\preccurlyeq}$ for a fixed value $a \in \mathbb{R}$

relations $L_{(\alpha, \beta)}^{\ll}$ and $L_{(\alpha, \beta)}^{\preccurlyeq}$ defined for $a$ and $b$ in $\mathbb{R}$ as

$$
L_{(\alpha, \beta)}^{\ll}(a, b)=\left\{\begin{array}{cl}
1 & \text { if } b-a>\alpha+\beta \\
0 & \text { if } b-a \leq \alpha \\
\frac{b-a-\alpha}{\beta} & \text { otherwise }(\beta>0)
\end{array}\right.
$$

expressing the degree to which $a$ is long before $b$, and

$$
L_{(\alpha, \beta)}^{\preccurlyeq}(a, b)=\left\{\begin{array}{cl}
1 & \text { if } b-a \geq-\alpha \\
0 & \text { if } b-a<-\alpha-\beta \\
\frac{b-a+\alpha+\beta}{\beta} & \text { otherwise }(\beta>0)
\end{array}\right.
$$

expressing the degree to which $a$ is before or at approximately the same time as $b$. The role of $\alpha$ and $\beta$ is illustrated in Figure 1. In both cases we assume $\beta \geq 0$ and $\alpha \in \mathbb{R}$. Our interpretation of the concepts long before and before or at approximately the same time as depends largely on the values of the parameters $\alpha$ and $\beta$. If $\alpha=\beta=0$, then $L_{(\alpha, \beta)}^{\ll}$ degenerates to $<$, and $L_{(\alpha, \beta)}^{\preccurlyeq}$ degenerates to $\leq$. On the other hand, when $\beta>0$ we have a gradual (linear) transition between satisfying and not satisfying these concepts.

It is easy to see that

$$
L_{(\alpha, \beta)}^{\preccurlyeq}(a, b)=1-L_{(\alpha, \beta)}^{\ll}(b, a)
$$

Recall that the (sup- $T$ ) composition $R \circ_{T} S$ of a fuzzy relation $R$ from $\mathbb{R}$ to $\mathbb{R}$ and a fuzzy relation $S$ from $\mathbb{R}$ to $\mathbb{R}$ is defined for $a$ and $c$ in $\mathbb{R}$ as

$$
\left(R \circ_{T} S\right)(a, c)=\sup _{b \in \mathbb{R}} T(R(a, b), S(b, c))
$$

where $T$ is an arbitrary $\mathrm{t}$-norm. In the following, we will use the Łukasiewicz t-norm $T_{w}$, and its dual t-conorm $S_{w}$, defined for all $a$ and $b$ in $[0,1]$ as

$$
\begin{aligned}
& T_{w}(a, b)=\max (0, a+b-1) \\
& S_{w}(a, b)=\min (1, a+b)
\end{aligned}
$$

For convenience, we write $R \circ S$ instead of $R \circ_{T_{w}} S$ in the remainder of this paper. We can prove the following characterizations of the compositions of $L_{(\alpha, \beta)}^{\ll}$ and $L_{(\alpha, \beta)}^{\preccurlyeq}$.

Proposition 1 (Composition): [11] Let $\alpha_{1}, \alpha_{2} \in \mathbb{R}$ and $\beta_{1}, \beta_{2} \geq 0$; it holds that

$$
\begin{aligned}
& L_{\left(\alpha_{1}, \beta_{1}\right)}^{\ll} \circ L_{\left(\alpha_{2}, \beta_{2}\right)}^{\ll}=L_{\left(\alpha_{1}+\alpha_{2}+\min \left(\beta_{1}, \beta_{2}\right), \max \left(\beta_{1}, \beta_{2}\right)\right)}^{\ll} \\
& L_{\left(\alpha_{1}, \beta_{1}\right)}^{\ll} \circ L_{\left(\alpha_{2}, \beta_{2}\right)}^{\preccurlyeq}=L_{\left(\alpha_{1}+\alpha_{2}, \max \left(\beta_{1}, \beta_{2}\right)\right)}^{\preccurlyeq} \\
& L_{\left(\alpha_{1}, \beta_{1}\right)}^{\preccurlyeq} \circ L_{\left(\alpha_{2}, \beta_{2}\right)}^{\ll}=L_{\left(\alpha_{2}-\alpha_{1}+\min \left(\beta_{1}, \beta_{2}\right)-\beta_{1}, \max \left(\beta_{1}, \beta_{2}\right)\right)}^{\ll} \\
& L_{\left(\alpha_{1}, \beta_{1}\right)}^{\ll} \circ L_{\left(\alpha_{2}, \beta_{2}\right)}^{\preccurlyeq}=L_{\left(\alpha_{1}-\alpha_{2}+\min \left(\beta_{1}, \beta_{2}\right)-\beta_{2}, \max \left(\beta_{1}, \beta_{2}\right)\right)}^{\ll}
\end{aligned}
$$

Note that in particular when $\alpha_{1}=\alpha_{2}=\beta_{1}=\beta_{2}=0$, this proposition corresponds to the transitivity rules for the crisp relations $<$ and $\leq$.

\section{B. Fuzzy time intervals}

We represent the time span of vague events as convex and upper semicontinuous normalised fuzzy sets in $\mathbb{R}$, which we call fuzzy (time) intervals. Recall that a fuzzy set $A$ in $\mathbb{R}$ with a bounded support is convex and upper semicontinuous iff for each $\alpha$ in $] 0,1]$ the $\alpha$-level set $\{x \mid A(x) \geq \alpha\}$ is a closed interval. If $A$ and $B$ are fuzzy time intervals, we cannot make use of boundary points to define (vague) temporal relations, since the boundaries of fuzzy time intervals may be imprecise. Instead we propose the definitions that are shown in the second column of Table I, where $I_{w}$ denotes the Łukasiewicz implicator, defined for $a$ and $b$ in $[0,1]$ by

$$
I_{w}(a, b)=\min (1,1-a+b)
$$

and $T_{w}$ is the Eukasiewicz $\mathrm{t}-$ norm as defined above. For example $b b_{(\alpha, \beta)}^{\ll}(A, B)$ expresses the degree to which the beginning of $A$ is long before the beginning of $B$, while $e b_{(\alpha, \beta)}^{\preccurlyeq}(A, B)$ expresses the degree to which the end of $A$ is before or at approximately the same time as the beginning of $B$. If there is no cause for confusion, we sometimes omit the subscript $(\alpha, \beta)$. It can be shown that if $A$ and $B$ are crisp intervals and $\alpha=\beta=0$, these definitions coincide with the corresponding definitions for crisp intervals, which are shown in the first column of Table I. Furthermore, (generalizations of) the transitivity properties of the crisp interval relations hold for these generalized definitions [11].

Consider for example the fuzzy time intervals $A$ and $B$ that are depicted in Figure 2. Table II shows the degree to which the eight fuzzy temporal relations from Table I are satisfied for different values of the parameters $\alpha$ and $\beta$. In general, we have that increasing the values of $\alpha$ and $\beta$ causes an increase (resp. a decrease) in the degree to which the fuzzy relations involving $L^{\preccurlyeq}$ (resp. $L^{\ll}$ ) are satisfied.

The main problem with the definitions of the fuzzy temporal relations is that it is unclear how to evaluate them in practice as they involve suprema and infima ranging over the real line. The most obvious solution is to apply discretization techniques, but these are computationally expensive. Assume 
TABLE I

DEFINITION OF THE QUALITATIVE TEMPORAL RELATIONS BETWEEN FUZZY TIME INTERVALS $A$ AND $B$, AND THEIR CORRESPONDENCE WITH THE CLASSiCAL DEFINITIONS WHEN $A=\left[a^{-}, a^{+}\right]$AND $B=\left[b^{-}, b^{+}\right]$ARE CRISP INTERVALS.

\begin{tabular}{|c|c|c|c|c|c|}
\hline \multicolumn{3}{|r|}{ Crisp intervals } & \multicolumn{3}{|r|}{ Fuzzy time intervals } \\
\hline$a^{-}<b^{-}$ & $\Leftrightarrow$ & $(\exists x)(x \in A \wedge(\forall y)(y \in B \Rightarrow x<y))$ & $b b_{(\alpha, \beta)}^{\ll}(A, B)$ & $=$ & $\sup _{x} T_{w}\left(A(x), \inf _{y} I_{w}\left(B(y), L_{(\alpha, \beta)}^{\ll}(x, y)\right)\right)$ \\
\hline$a^{-} \leq b^{-}$ & $\Leftrightarrow$ & $(\forall y)(y \in B \Rightarrow(\exists x)(x \in A \wedge x \leq y))$ & $b b_{(\alpha, \beta)}^{\preccurlyeq}(A, B)$ & $=$ & $\inf _{y} I_{w}\left(B(y), \sup _{x} T_{w}\left(A(x), L_{(\alpha, \beta)}^{\preccurlyeq}(x, y)\right)\right)$ \\
\hline$a^{+}<b^{+}$ & $\Leftrightarrow$ & $(\exists y)(y \in B \wedge(\forall x)(x \in A \Rightarrow x<y))$ & $e e_{(\alpha, \beta)}^{\ll}(A, B)$ & $=$ & $\sup _{y} T_{w}\left(B(y), \inf _{x} I_{w}\left(A(x), L_{(\alpha, \beta)}^{\ll}(x, y)\right)\right)$ \\
\hline$a^{+} \leq b^{+}$ & $\Leftrightarrow$ & $(\forall x)(x \in A \Rightarrow(\exists y)(y \in B \wedge x \leq y))$ & $e e_{(\alpha, \beta)}(A, B)$ & $=$ & $\inf _{x} I_{w}\left(A(x), \sup _{y} T_{w}\left(B(y), L_{(\alpha, \beta)}(x, y)\right)\right)$ \\
\hline$a^{+}<b^{-}$ & $\Leftrightarrow$ & $(\forall x)(\forall y)(x \in A \wedge y \in B \Rightarrow x<y)$ & $e b_{(\alpha, \beta)}^{\ll}(A, B)$ & $=$ & $\inf _{x} I_{w}\left(A(x), \inf _{y} I_{w}\left(B(y), L_{(\alpha, \beta)}^{\ll}(x, y)\right)\right)$ \\
\hline$a^{+} \leq b^{-}$ & $\Leftrightarrow$ & $(\forall x)(\forall y)(x \in A \wedge y \in B \Rightarrow x \leq y)$ & $e b_{(\alpha, \beta)}^{\preccurlyeq}(A, B)$ & $=$ & $\inf _{x} I_{w}\left(A(x), \inf _{y} I_{w}\left(B(y), L_{(\alpha, \beta)}^{\preccurlyeq}(x, y)\right)\right)$ \\
\hline$a^{-}<b^{+}$ & $\Leftrightarrow$ & $(\exists x)(\exists y)(x \in A \wedge y \in B \wedge x<y)$ & $b e_{(\alpha, \beta)}^{\ll}(A, B)$ & $=$ & $\sup _{x} T_{w}\left(A(x), \sup _{y} T_{w}\left(B(y), L_{(\alpha, \beta)}^{\ll}(x, y)\right)\right)$ \\
\hline$a^{-} \leq b^{+}$ & $\Leftrightarrow$ & $(\exists x)(\exists y)(x \in A \wedge y \in B \wedge x \leq y)$ & $b e_{(\alpha, \beta)}^{\preccurlyeq}(A, B)$ & $=$ & $\sup _{x} T_{w}\left(A(x), \sup _{y} T_{w}\left(B(y), L_{(\alpha, \beta)}^{\preccurlyeq}(x, y)\right)\right)$ \\
\hline
\end{tabular}

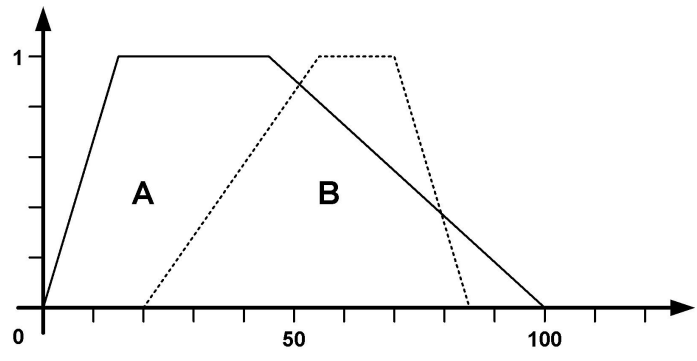

Fig. 2. Two fuzzy time intervals $A$ and $B$

for example that we choose somehow $n$ points $x_{i}$ and $n$ points $y_{j}(1 \leq i, j \leq n)$ such that

$$
\max _{i=1}^{n} T_{w}\left(A\left(x_{i}\right), \max _{j=1}^{n} T_{w}\left(B\left(y_{j}\right), L^{\preccurlyeq}\left(x_{i}, x_{j}\right)\right)\right)
$$

is a reasonable approximation of $b e^{\preccurlyeq}(A, B)$. Evaluating (2) still requires $\Theta\left(n^{2}\right)$ basic arithmetic operations. In the next section we provide an efficient characterization of the fuzzy temporal relations that is exact for piecewise linear fuzzy intervals. Moreover, this characterization can be used to approximate the fuzzy temporal relations for arbitrary fuzzy time intervals in a way that is much more efficient (i.e. kwadratic in the number of line segments that are used to approximate the fuzzy time intervals).

TABLE II

EVALUATION OF THE FUZZY TEMPORAL RELATIONS FOR THE FUZZY INTERVALS $A$ AND $B$ FROM FIGURE 2 FOR DIFFERENT VALUES OF THE PARAMETERS $(\alpha, \beta)$

\begin{tabular}{|c|c|c|c|c|c|c|}
\hline$(\alpha, \beta)$ & $(0,0)$ & $(0,20)$ & $(20,0)$ & $(20,20)$ & $(40,0)$ & $(40,20)$ \\
\hline$b b^{\ll}(A, B)$ & 1 & 1 & 0.58 & 0.57 & 0 & 0 \\
\hline$b b^{\preccurlyeq}(A, B)$ & 1 & 1 & 1 & 1 & 1 & 1 \\
\hline$e e^{\ll}(A, B)$ & 0.45 & 0.45 & 0.09 & 0.09 & 0 & 0 \\
\hline$e e^{\preccurlyeq}(A, B)$ & 0.73 & 0.81 & 1 & 1 & 1 & 1 \\
\hline$e b^{\ll}(A, B)$ & 0.18 & 0.18 & 0 & 0 & 0 & 0 \\
\hline$e b^{\preccurlyeq}(A, B)$ & 0.18 & 0.55 & 0.55 & 0.91 & 0.91 & 1 \\
\hline$b e^{\ll}(A, B)$ & 1 & 1 & 1 & 1 & 1 & 0.75 \\
\hline$b e^{\preccurlyeq}(A, B)$ & 1 & 1 & 1 & 1 & 1 & 1 \\
\hline
\end{tabular}

\section{A CHARACTERIZATION FOR PIECEWISE LINEAR FUZZY INTERVALS}

\section{A. Piecewise linear fuzzy intervals}

In the following, we use $A=\left(\left(a_{0} / \lambda_{0}\right),\left(a_{1} / \lambda_{1}\right)\right)$, where $a_{0} \leq a_{1}$ and $\lambda_{0}, \lambda_{1}$ in $[0,1]$, to denote a linear fuzzy set in $\mathbb{R}$, i.e. for each $x$ in $\mathbb{R}$, we have

$$
A(x)= \begin{cases}\lambda_{0}+\left(x-a_{0}\right) \frac{\lambda_{1}-\lambda_{0}}{a_{1}-a_{0}} & \text { if } a_{0} \leq x<a_{1} \\ \lambda_{1} & \text { if } x=a_{1} \\ 0 & \text { otherwise }\end{cases}
$$

Note that when $a_{0}=a_{1}$ and $\lambda_{1}>0, A$ degenerates to a fuzzy singleton and the value $\lambda_{0}$ is ignored. Every piecewise linear fuzzy interval $A$ is equal to the union of a finite set of linear fuzzy sets, where the union of two fuzzy sets $A$ and $B$ in $\mathbb{R}$ is defined as $(A \cup B)(x)=\max (A(x), B(x))$ for all $x$ in $\mathbb{R}$. Due to the following two propositions, we only need to provide a characterization for linear fuzzy sets.

Proposition 2: Let $A=\bigcup_{i=1}^{n} A_{i}, B=\bigcup_{j=1}^{m} B_{j}, A_{i}=$ $\left(\left(a_{0}^{i} / \lambda_{0}^{i}\right),\left(a_{1}^{i} / \lambda_{1}^{i}\right)\right)$ and $B_{j}=\left(\left(b_{0}^{j} / \delta_{0}^{j}\right),\left(b_{1}^{j} / \delta_{1}^{j}\right)\right)$ for $1 \leq$ $i \leq n$ and $1 \leq j \leq m$. It holds that

$$
\begin{aligned}
e b^{\ll}(A, B) & =\min _{i=1}^{n} \min _{j=1}^{m} e b^{\ll}\left(A_{i}, B_{j}\right) \\
e b^{\preccurlyeq}(A, B) & =\min _{i=1}^{n} \min _{j=1}^{m} e b^{\preccurlyeq}\left(A_{i}, B_{j}\right) \\
b e^{\ll}(A, B) & =\max _{i=1} \max _{j=1} b e^{\ll}\left(A_{i}, B_{j}\right) \\
b e^{\preccurlyeq}(A, B) & =\max _{i=1}^{n} \max _{j=1}^{m} b e^{\preccurlyeq}\left(A_{i}, B_{j}\right)
\end{aligned}
$$

Proof: As an example we prove the first equality. We have that

$$
\begin{aligned}
& e b^{\ll}(A, B) \\
& =\inf _{x} I_{w}\left(A(x), \inf _{y} I_{w}\left(B(y), L^{\ll}(x, y)\right)\right) \\
& =\inf _{x} I_{w}\left(\max _{i=1}^{n} A_{i}(x), \inf _{y} I_{w}\left(\max _{j=1}^{m} B_{j}(y), L^{\ll}(x, y)\right)\right) \\
& =\inf _{x} \min _{i=1}^{n} I_{w}\left(A_{i}(x), \inf _{y} \min _{j=1}^{m} I_{w}\left(B_{j}(y), L^{\ll}(x, y)\right)\right) \\
& =\min _{i=1}^{n} \inf _{x} I_{w}\left(A_{i}(x), \min _{j=1} \inf _{y} I_{w}\left(B_{j}(y), L^{\ll}(x, y)\right)\right)
\end{aligned}
$$




$$
\begin{aligned}
& =\min _{i=1}^{n} \min _{j=1}^{m} \inf _{x} I_{w}\left(A_{i}(x), \inf _{y} I_{w}\left(B_{j}(y), L^{\ll}(x, y)\right)\right) \\
& =\min _{i=1}^{n} \min _{j=1} e b^{\ll}\left(A_{i}, B_{j}\right)
\end{aligned}
$$

Note that the proof of Proposition 2 is independent of the fact that the relations $L^{\ll}$ and $L^{\preccurlyeq}$ are used, e.g. for an arbitrary fuzzy relation $S$ in $\mathbb{R}$ we also have that

$$
\begin{aligned}
\inf _{x} & I_{w}\left(A(x), \inf _{y} I_{w}(B(y), S(x, y))\right) \\
& =\min _{i=1}^{n} \min _{j=1}^{m} \inf _{x} I_{w}\left(A_{i}(x), \inf _{y} I_{w}\left(B_{j}(y), S(x, y)\right)\right)
\end{aligned}
$$

Unfortunately, a similar characterization for $b b^{\ll}(A, B)$, $b b^{\preccurlyeq}(A, B), e e^{\ll}(A, B)$, and $e e^{\preccurlyeq}(A, B)$ is not valid. Note that a proof analogous to the proof of Proposition 2 is not possible, since in general

$$
\begin{gathered}
\inf _{i} \max \left(x_{i}, y_{i}\right) \neq \max \left(\inf _{i} x_{i}, \inf _{i} y_{i}\right) \\
\sup _{i} \min \left(x_{i}, y_{i}\right) \neq \min \left(\sup _{i} x_{i}, \sup _{i} y_{i}\right)
\end{gathered}
$$

To obtain characterizations of the remaining fuzzy temporal relations, we will use the following lemma.

Lemma 1: Let $A=\left(\left(a_{0} / \lambda_{0}\right),\left(a_{1} / \lambda_{1}\right)\right)$, and $a_{0}<a_{1}$. Let $C(y)=\sup _{x} T_{w}\left(A(x), L_{(\alpha, \beta)}^{\preccurlyeq}(x, y)\right)$ for all $y$ in $\mathbb{R}$. Then $C$ is a piecewise linear fuzzy set. Furthermore, if $\lambda_{0}<\lambda_{1}$, for all $y$ in $\mathbb{R}$ it holds that

$$
\begin{aligned}
& \sup _{x} T_{w}\left(A(x), L_{(\alpha, \beta)}^{\preccurlyeq}(x, y)\right) \\
& = \begin{cases}0 & \text { if } y+\alpha<a_{0}-\lambda_{0} \beta \\
\frac{y-a_{0}+\alpha+\lambda_{0} \beta}{\beta} & \text { if } a_{0}-\lambda_{0} \beta \leq y+\alpha<a_{0} \\
A(y+\alpha) & \text { if } a_{0} \leq y+\alpha<a_{1} \\
\lambda_{1} & \text { if } a_{1} \leq y+\alpha\end{cases}
\end{aligned}
$$

provided $\frac{a_{1}-a_{0}}{\lambda_{1}-\lambda_{0}} \geq \beta$, and

$$
\begin{aligned}
& \sup _{x} T_{w}\left(A(x), L_{(\alpha, \beta)}^{\preccurlyeq}(x, y)\right) \\
& = \begin{cases}0 & \text { if } y+\alpha<a_{1}-\lambda_{1} \beta \\
\frac{y-a_{1}+\alpha+\lambda_{1} \beta}{\beta} & \text { if } a_{1}-\lambda_{1} \beta \leq y+\alpha<a_{1} \\
\lambda_{1} & \text { if } a_{1} \leq y+\alpha\end{cases}
\end{aligned}
$$

provided $\frac{a_{1}-a_{0}}{\lambda_{1}-\lambda_{0}} \leq \beta$. Moreover, if $\lambda_{0} \geq \lambda_{1}$, for all $y$ in $\mathbb{R}$ it holds that

$$
\begin{aligned}
& \sup _{x} T_{w}\left(A(x), L_{(\alpha, \beta)}^{\preccurlyeq}(x, y)\right) \\
& = \begin{cases}0 & \text { if } y+\alpha<a_{0}-\lambda_{0} \beta \\
\frac{y-a_{0}+\alpha+\lambda_{0} \beta}{\beta} & \text { if } a_{0}-\lambda_{0} \beta \leq y+\alpha<a_{0} \\
\lambda_{0} & \text { if } a_{0} \leq y+\alpha\end{cases}
\end{aligned}
$$

Example 1: Consider the linear fuzzy set $A_{1}$ in Figure 3(a). The corresponding piecewise linear fuzzy set $C_{1}$ defined for all $y$ in $\mathbb{R}$ by

$$
C_{1}(y)=\sup _{x} T_{w}\left(A(x), L_{(20,20)}^{\preccurlyeq}(x, y)\right)
$$

is shown in Figure 3(b).

Proposition 3: Let $A=\bigcup_{i=1}^{n} A_{i}, B=\bigcup_{j=1}^{m} B_{j}, A_{i}=$ $\left(\left(a_{0}^{i} / \lambda_{0}^{i}\right),\left(a_{1}^{i} / \lambda_{1}^{i}\right)\right)$ and $B_{j}=\left(\left(b_{0}^{j} / \delta_{0}^{j}\right),\left(b_{1}^{j} / \delta_{1}^{j}\right)\right)$ for $1 \leq$ $i \leq n$ and $1 \leq j \leq m$. We can always find linear fuzzy sets $A_{k}^{\prime}$ and $B_{l}^{\prime}\left(1 \leq k \leq n^{\prime}, 1 \leq l \leq m^{\prime}, n^{\prime} \geq n\right.$ and $\left.m^{\prime} \geq m\right)$ such that $A=\bigcup_{k=1}^{n^{\prime}} A_{k}^{\prime}, B=\bigcup_{l=1}^{m^{\prime}} B_{l}^{\prime}$ and

$$
\begin{aligned}
b b^{\ll}(A, B) & =\max _{k=1}^{n^{\prime}} \min _{j=1}^{m} b b^{\ll}\left(A_{k}^{\prime}, B_{j}\right) \\
b b^{\preccurlyeq}(A, B) & =\min _{l=1}^{m^{\prime}} \max _{i=1}^{n} b b^{\preccurlyeq}\left(A_{i}, B_{l}^{\prime}\right) \\
e e^{\ll}(A, B) & =\max _{l=1}^{m^{\prime}} \min _{i=1}^{n} e e^{\ll}\left(A_{i}, B_{l}^{\prime}\right) \\
e e^{\preccurlyeq}(A, B) & =\min _{k=1} \max _{j=1}^{m} e e^{\preccurlyeq}\left(A_{k}^{\prime}, B_{j}\right)
\end{aligned}
$$

Proof: As an example, we prove the second equality. The definition of $b b^{\preccurlyeq}(A, B)$ contains the formula

$$
\sup _{x} T_{w}\left(A(x), L_{(\alpha, \beta)}^{\preccurlyeq}(x, y)\right)
$$

which we abbreviate as $C(y)$ for all $y$ in $\mathbb{R}$. From Lemma 1 we know that $C$ is a piecewise linear fuzzy set. Furthermore, since $A=\bigcup_{i=1}^{n} A_{i}$, (3) equals

$$
\max _{i=1}^{n} \sup _{x} T_{w}\left(A_{i}(x), L_{(\alpha, \beta)}^{\preccurlyeq}(x, y)\right)
$$

According to Lemma 1 , for all $i$ in $\{1, \ldots, n\}$, the fuzzy sets $C_{i}$ defined as

$$
C_{i}(y)=\sup _{x} T_{w}\left(A_{i}(x), L_{(\alpha, \beta)}^{\preccurlyeq}(x, y)\right)
$$

are all piecewise linear fuzzy sets. From (3) equals (4) we derive that $C$ is the union of all $C_{i}$ 's $(i=1, \ldots, n)$. We now decompose each of the original linear fuzzy sets $B_{j}$ in one or more linear fuzzy sets $B_{l}^{\prime}$ of the form $B_{l}^{\prime}=$ $\left(\left({b^{\prime}}_{0}^{l} /{\delta^{\prime}}_{0}^{l}\right),\left({b^{\prime}}_{1}^{l} /{\delta^{\prime}}_{1}^{l}\right)\right)$. The parameters ${b^{\prime}}_{0}^{l}$ and ${b^{\prime}}_{1}^{l}$ are chosen such that there exists an $i_{l}$ in $\{1,2, \ldots, n\}$ with

$$
C(y)=C_{i_{l}}(y)
$$

for all $y$ in $\left[b^{\prime l}{ }_{0}, b_{1}^{l}{ }_{1}^{l}\right]$. Furthermore they are restricted by the fact that the line segment defining $B_{l}^{\prime}$ is a subsegment of the line segment that defines $B_{j}$. Obviously ${\delta^{\prime}}_{0}^{l}=B\left(b_{0}^{\prime l}\right)$ and ${\delta^{\prime}}^{l}{ }_{1}=B\left({b^{\prime}}_{1}^{l}\right)$. We obtain

$$
\begin{aligned}
& b b^{\preccurlyeq}(A, B) \\
& =\inf _{y} I_{w}\left(B(y), \sup _{x} T_{w}\left(A(x), L^{\preccurlyeq}(x, y)\right)\right) \\
& =\inf _{y} I_{w}\left(\max _{l=1}^{m^{\prime}} B_{l}^{\prime}(y), \sup _{x} T_{w}\left(A(x), L^{\preccurlyeq}(x, y)\right)\right) \\
& =\inf _{y} \min _{l=1}^{m^{\prime}} I_{w}\left(B_{l}^{\prime}(y), \sup _{x} T_{w}\left(A(x), L^{\preccurlyeq}(x, y)\right)\right) \\
& =\min _{l=1}^{m^{\prime}} \inf _{y} I_{w}\left(B_{l}^{\prime}(y), \sup _{x} T_{w}\left(A(x), L^{\preccurlyeq}(x, y)\right)\right)
\end{aligned}
$$

For $y \notin\left[{b^{\prime}}_{0}^{l},{b^{\prime}}_{1}^{l}\right]$, we have that $B_{l}^{\prime}(y)=0$, and as a consequence

$$
I_{w}\left(B_{l}^{\prime}(y), \sup _{x} T_{w}\left(A(x), L^{\preccurlyeq}(x, y)\right)\right)=1
$$


hence we obtain for certain $A_{i_{l}}$ 's

$$
\begin{aligned}
& b b^{\preccurlyeq}(A, B) \\
& =\min _{l=1}^{m^{\prime}} \inf _{y} I_{w}\left(B_{l}^{\prime}(y), \sup _{x} T_{w}\left(A(x), L^{\preccurlyeq}(x, y)\right)\right) \\
& =\min _{l=1}^{m^{\prime}} \inf _{y \in\left[b_{0}^{\prime l}, b^{\prime l}{ }_{1}^{l}\right]} I_{w}\left(B_{l}^{\prime}(y), \sup _{x} T_{w}\left(A(x), L^{\preccurlyeq}(x, y)\right)\right) \\
& =\min _{l=1}^{m^{\prime}} \inf _{y \in\left[b^{\prime}{ }_{0}, b^{\prime}{ }_{1}\right]} I_{w}\left(B_{l}^{\prime}(y), \sup _{x} T_{w}\left(A_{i_{l}}(x), L^{\preccurlyeq}(x, y)\right)\right) \\
& \leq \min _{l=1} \max _{i=1} \inf _{y \in\left[b_{0}^{\prime}{ }_{0}, b_{1}^{\prime}{ }_{1}\right]} I_{w}\left(B_{l}^{\prime}(y), \sup _{x} T_{w}\left(A_{i}(x), L^{\preccurlyeq}(x, y)\right)\right)
\end{aligned}
$$

Hence

$$
\begin{aligned}
& b b^{\preccurlyeq}(A, B) \\
& \leq \min _{l=1}^{m^{\prime}} \max _{i=1}^{n} \inf _{y} I_{w}\left(B_{l}^{\prime}(y), \sup _{x} T_{w}\left(A_{i}(x), L^{\preccurlyeq}(x, y)\right)\right) \\
& =\min _{l=1} \max _{i=1}^{n} b b^{\preccurlyeq}\left(A_{i}, B_{l}^{\prime}\right)
\end{aligned}
$$

Conversely we find

$$
\begin{aligned}
& \min _{l=1}^{m^{\prime}} \max _{i=1}^{n} b b^{\preccurlyeq}\left(A_{i}, B_{l}^{\prime}\right) \\
& =\min _{l=1}^{m^{\prime}} \max _{i=1}^{n} \inf _{y \in\left[b_{0}^{\prime}, b_{1}^{\prime}\right]} I_{w}\left(B_{l}^{\prime}(y)\right. \\
& \left.\sup _{x} T_{w}\left(A_{i}(x), L^{\preccurlyeq}(x, y)\right)\right) \\
& \leq \min _{l=1}^{m^{\prime}} \max _{i=1}^{n} \inf _{y \in\left[b_{0}^{\prime}, b_{1}^{\prime l}\right]} I_{w}\left(B_{l}^{\prime}(y)\right. \\
& \left.\sup _{x} T_{w}\left(\max _{i^{\prime}=1}^{n} A_{i^{\prime}}(x), L^{\preccurlyeq}(x, y)\right)\right) \\
& =\min _{l=1}^{m^{\prime}} \max _{i=1}^{n} \inf _{y \in\left[b_{0}^{\prime}, b_{1}^{\prime l}\right]} I_{w}\left(B_{l}^{\prime}(y)\right. \\
& \left.\sup _{x} T_{w}\left(A(x), L^{\preccurlyeq}(x, y)\right)\right) \\
& =\min _{l=1}^{m^{\prime}} \max _{i=1}^{n} \inf _{y \in\left[b_{0}^{\prime}, b_{1}^{\prime}\right]} I_{w}\left(B_{l}^{\prime}(y)\right. \\
& \left.\sup _{x} T_{w}\left(A_{i_{l}}(x), L^{\preccurlyeq}(x, y)\right)\right) \\
& =\min _{l=1}^{m^{\prime}} \inf _{y \in\left[b_{0}^{\prime}, b_{1}^{\prime}{ }_{1}^{l}\right]} I_{w}\left(B_{l}^{\prime}(y), \sup _{x} T_{w}\left(A_{i_{l}}(x), L^{\preccurlyeq}(x, y)\right)\right) \\
& =b b^{\preccurlyeq}(A, B)
\end{aligned}
$$

Example 2: Consider the piecewise linear fuzzy intervals in figure 3(a), and assume that we want to evaluate $b b_{(20,20)}^{\preccurlyeq}(A, B)$ using Proposition 3. To find out how the linear fuzzy sets $B_{1}, B_{2}$ and $B_{3}$ should be decomposed we apply Lemma 1 to obtain a representation as a piecewise

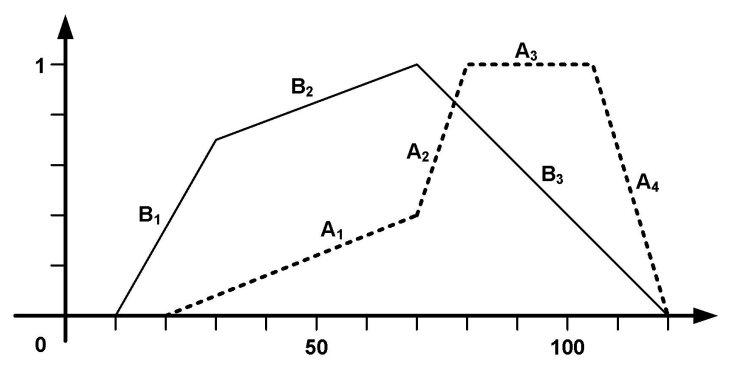

(a) Initial decomposition of $A$ and $B$ into linear fuzzy sets

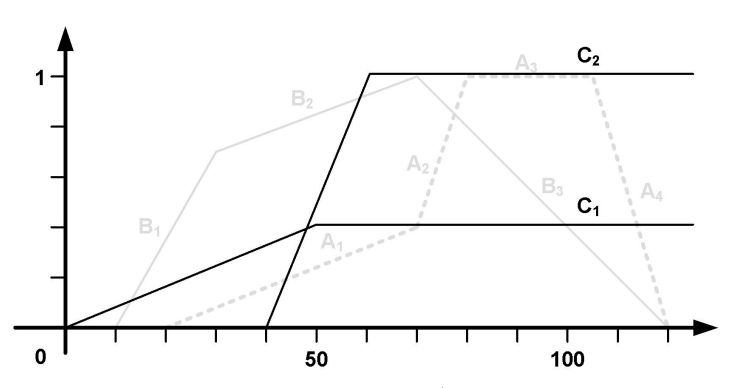

(b) $\sup _{x} T_{w}\left(A_{i}(x), L_{(\alpha, \beta)}^{\preccurlyeq}(x, y)\right)$

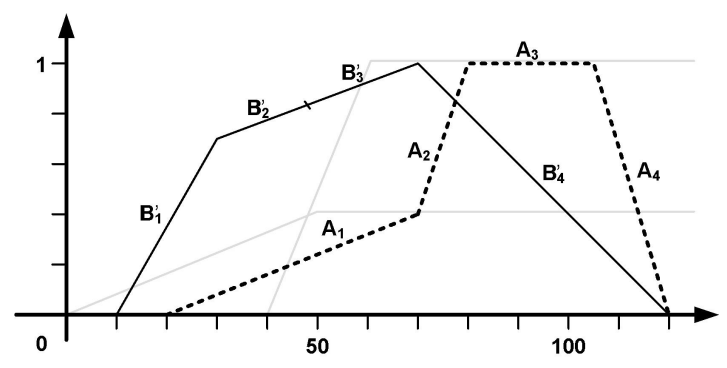

(c) New decompostion of $B$ into linear fuzzy sets

Fig. 3. Obtaining new line segments for $B$ to apply Proposition 3.

linear fuzzy set for

$$
\begin{aligned}
& C_{1}(y)=\sup _{x} T_{w}\left(A_{1}(x), L_{(20,20)}^{\preccurlyeq}(x, y)\right) \\
& C_{2}(y)=\sup _{x} T_{w}\left(A_{2}(x), L_{(20,20)}^{\preccurlyeq}(x, y)\right) \\
& C_{3}(y)=\sup _{x} T_{w}\left(A_{3}(x), L_{(20,20)}^{\preccurlyeq}(x, y)\right) \\
& C_{4}(y)=\sup _{x} T_{w}\left(A_{4}(x), L_{(20,20)}^{\preccurlyeq}(x, y)\right)
\end{aligned}
$$

$C_{1}$ and $C_{2}$ are depicted in Figure 3(b). Furthermore, it holds that $C_{3}(y)=C_{2}(y)$ and $C_{4}(y)=C_{3}(y-25) \leq C_{3}(y)$ for all $y \in \mathbb{R}$. We have that

$$
\sup _{x} T_{w}\left(A(x), L_{(20,20)}^{\preccurlyeq}(x, y)\right)= \begin{cases}C_{1}(y) & \text { for all } y \leq \frac{1000}{21} \\ C_{2}(y) & \text { for all } y \geq \frac{1000}{21}\end{cases}
$$

From this we can deduce that the line segment defining $B_{1}$ should not be divided into subsegments, as for all $y \in[10,30]$, it holds that $C(y)=C_{1}(y)$. Neither should the line segment defining $B_{3}$ be divided into subsegments, as for all $y \in[70,120]$ it holds that $C(y)=C_{2}(y)$. However, $B_{2}$ should be decomposed into the linear fuzzy sets $B_{2}^{\prime}=\left((30 / 0),\left(\frac{1000}{21} / \frac{8}{21}\right)\right)$ and $B_{3}^{\prime}=\left(\left(\frac{1000}{21} / \frac{8}{21}\right),(70 / 1)\right)$, 


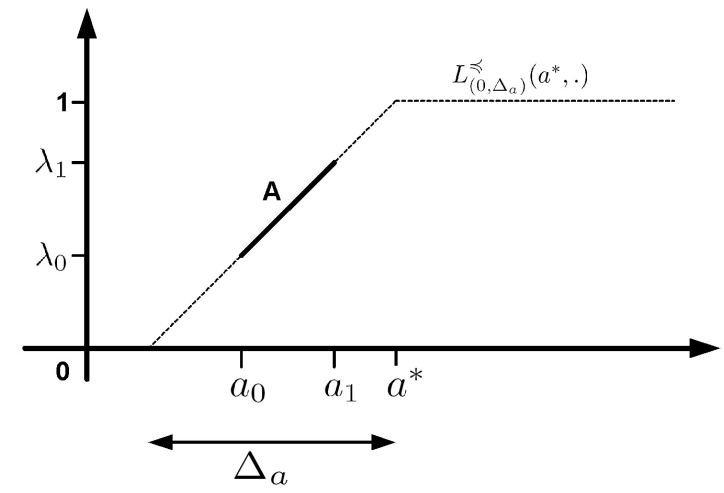

Fig. 4. If $A=\left(\left(a_{0} / \lambda_{0}\right),\left(a_{1} / \lambda_{1}\right)\right)$ and $\lambda_{0}<\lambda_{1}$ it holds that $A(x)=$ $L_{\left(0, \Delta_{a}\right)}^{\preccurlyeq}\left(a^{*}, x\right)$, for $x \in\left[a_{0}, a_{1}\right]$

as $C(y)=C_{1}(y)$ for $y \in\left[30, \frac{1000}{21}\right]$, and $C(y)=C_{2}(y)$ for $y \in\left[\frac{1000}{21}, 70\right]$.

\section{B. Linear fuzzy intervals}

Because of Proposition 2 and Proposition 3, the problem of finding a characterization for piecewise linear fuzzy time intervals is reduced to finding a characterization for linear fuzzy time intervals. In the remainder of this section, we will show how $b e^{\preccurlyeq}(A, B)$ can be evaluated when $A$ and $B$ are linear fuzzy intervals. Similar results can be obtained for the other fuzzy temporal relations from Table I. Due to limited space, we omit these results here.

Proposition 4: Let $A=\left(\left(a_{0} / \lambda_{0}\right),\left(a_{1} / \lambda_{1}\right)\right), \lambda_{0}, \lambda_{1} \in$ $[0,1], a_{0}<a_{1}, B=\left(\left(b_{0} / \delta_{0}\right),\left(b_{1} / \delta_{1}\right)\right), \delta_{0}, \delta_{1} \in[0,1]$, $b_{0}<b_{1}$. Furthermore let $\Delta_{a}$ and $\Delta_{b}$ be defined as follows:

$$
\begin{aligned}
& \Delta_{a}= \begin{cases}\frac{a_{1}-a_{0}}{\lambda_{1}-\lambda_{0}} & \text { if } \lambda_{1}>\lambda_{0} \\
0 & \text { otherwise }\end{cases} \\
& \Delta_{b}= \begin{cases}\frac{b_{1}-b_{0}}{\delta_{0}-\delta_{1}} & \text { if } \delta_{0}>\delta_{1} \\
0 & \text { otherwise }\end{cases}
\end{aligned}
$$

The characterization of $b e^{\preccurlyeq}(A, B)$ depends on the relative ordering of $\Delta_{a}, \Delta_{b}$ and $\beta$. The results are summarized in Table III.

Proof: (sketch) First assume that $\lambda_{0}<\lambda_{1}$ and $\delta_{0}>\delta_{1}$. It then holds that $A(x)=L_{\left(0, \Delta_{a}\right)}^{\preccurlyeq}\left(a^{*}, x\right)$ for all $x \in\left[a_{0}, a_{1}\right]$, where

$$
a^{*}=a_{1}+\frac{\left(1-\lambda_{1}\right)\left(a_{1}-a_{0}\right)}{\lambda_{1}-\lambda_{0}}
$$

This is illustrated in Figure 4. In the same way we have that $B(y)=L_{\left(0, \Delta_{b}\right)}^{\preccurlyeq}\left(y, b^{*}\right)$ for all $y \in\left[b_{0}, b_{1}\right]$, where

$$
b^{*}=b_{0}-\frac{\left(1-\delta_{0}\right)\left(b_{1}-b_{0}\right)}{\delta_{0}-\delta_{1}}
$$

Thus we obtain that

$$
\begin{aligned}
& b e^{\preccurlyeq}(A, B) \\
& =\sup _{x} T_{w}\left(A(x), \sup _{y} T_{w}\left(B(y), L_{(\alpha, \beta)}^{\preccurlyeq}(x, y)\right)\right) \\
& =\sup _{x \in\left[a_{0}, a_{1}\right]} T_{w}\left(L_{\left(0, \Delta_{a}\right)}^{\preccurlyeq}\left(a^{*}, x\right),\right. \\
& \left.\sup _{y \in\left[b_{0}, b_{1}\right]} T_{w}\left(L_{\left(0, \Delta_{b}\right)}^{\preccurlyeq}\left(y, b^{*}\right), L_{(\alpha, \beta)}^{\preccurlyeq}(x, y)\right)\right)
\end{aligned}
$$

It can be shown that in general $\left(y_{0} \leq y_{1}\right)$

$$
\begin{gathered}
\sup _{y \in\left[y_{0}, y_{1}\right]} T_{w}\left(L_{\left(\alpha_{1}, \beta_{1}\right)}^{\preccurlyeq}(x, y), L_{\left(\alpha_{2}, \beta_{2}\right)}^{\preccurlyeq}(y, z)\right) \\
=T_{w}\left(L_{\left(\alpha_{1}, \beta_{1}\right)}^{\preccurlyeq}\left(x, \min \left(y_{1}, \max \left(y_{0}, z+\alpha_{2}\right)\right)\right),\right. \\
\left.L_{\left(\alpha_{2}, \beta_{2}\right)}^{\preccurlyeq}\left(y_{0}, z\right)\right)
\end{gathered}
$$

provided $\beta_{1} \geq \beta_{2}$, and

$$
\begin{aligned}
\sup _{y \in\left[y_{0}, y_{1}\right]} & T_{w}\left(L_{\left(\alpha_{1}, \beta_{1}\right)}^{\preccurlyeq}(x, y), L_{\left(\alpha_{2}, \beta_{2}\right)}^{\preccurlyeq}(y, z)\right) \\
= & T_{w}\left(L_{\left(\alpha_{1}, \beta_{1}\right)}^{\preccurlyeq}\left(x, y_{1}\right),\right. \\
& \left.L_{\left(\alpha_{2}, \beta_{2}\right)}^{\preccurlyeq}\left(\min \left(y_{1}, \max \left(y_{0}, x-\alpha_{1}\right)\right), z\right)\right)
\end{aligned}
$$

provided $\beta_{1} \leq \beta_{2}$. To complete the proof, we can apply (10) and (11) to (9). This last part of the proof is more or less straightforward, but tedious as it involves a case analysis on the relative ordering of $\Delta_{a}, \Delta_{b}$ and $\beta$, as well as on the relative ordering of $a_{0}, a_{1}, b_{0}+\alpha$, and $b_{1}$.

Now assume that $\lambda_{0} \geq \lambda_{1}$ and $\delta_{0}>\delta_{1}$. It holds that

$$
\begin{aligned}
& b e^{\preccurlyeq}(A, B) \\
& =\sup _{x} T_{w}\left(A(x), \sup _{y} T_{w}\left(B(y), L_{(\alpha, \beta)}^{\preccurlyeq}(x, y)\right)\right) \\
& =T_{w}\left(\lambda_{0}, \sup _{y} T_{w}\left(B(y), L_{(\alpha, \beta)}^{\preccurlyeq}\left(a_{0}, y\right)\right)\right) \\
& =T_{w}\left(\lambda_{0}, \sup _{y \in\left[b_{0}, b_{1}\right]} T_{w}\left(L_{\left(0, \Delta_{b}\right)}^{\preccurlyeq}\left(y, b^{*}\right), L_{(\alpha, \beta)}^{\preccurlyeq}\left(a_{0}, y\right)\right)\right)
\end{aligned}
$$

and again we can apply (10) or (11). The proof for $\delta_{0} \leq \delta_{1}$ is analogous.

Although the characterization in Table III is more longwinded than the corresponding definition in Table I, it makes implementing the fuzzy temporal relations very straightforward. Moreover, the time complexity of evaluating the fuzzy temporal relations for linear fuzzy intervals is constant. Because of Proposition 2 and Proposition 3, the time complexity of evaluating the fuzzy temporal relations for two piecewise linear fuzzy sets $A$ and $B$ is kwadratic in the number of line segments that are used to define $A$ and $B$. Note that in practice it is unlikely that more than a few line segments are used to define a fuzzy time interval.

\section{A CHARACTERIZATION FOR TRAPEZOIDALLY SHAPED FUZZY SETS}

In this section we discuss the behaviour of a special class of piecewise linear fuzzy intervals, namely trapezoidally shaped fuzzy sets. In particular, we will show that a more 
TABLE III

CHARACTERIZATION OF THE FUZZY TEMPORAL RELATION $b e^{\preccurlyeq}$ FOR LINEAR FUZZY TIME INTERVALS $A$ AND $B$

\begin{tabular}{|c|l|}
\hline Sufficient condition & Characterization \\
\hline$\beta<\Delta_{b} \leq \Delta_{a}$ & $b e^{\preccurlyeq}(A, B)=T_{w}\left(\lambda_{1}, \delta_{0}, L_{\left(0, \Delta_{a}\right)}^{\preccurlyeq}\left(a_{1}, \max \left(b_{0}+\alpha, a_{0}\right)\right), L_{\left(0, \Delta_{b}\right)}^{\preccurlyeq}\left(a_{0}, \max \left(b_{0}+\alpha, a_{0}-b_{1}+b_{0}\right)\right), L_{(\alpha, \beta)}^{\preccurlyeq}\left(a_{0}, b_{1}\right)\right)$ \\
$\beta<\Delta_{a} \leq \Delta_{b}$ & $b e^{\preccurlyeq}(A, B)=T_{w}\left(\lambda_{1}, \delta_{0}, L_{\left(0, \Delta_{b}\right)}^{\preccurlyeq}\left(\min \left(a_{1}-\alpha, b_{1}\right), b_{0}\right), L_{\left(0, \Delta_{a}\right)}^{\preccurlyeq}\left(\min \left(a_{1}-\alpha, b_{1}+a_{1}-a_{0}\right), b_{1}\right), L_{(\alpha, \beta)}^{\preccurlyeq}\left(a_{0}, b_{1}\right)\right)$ \\
$0<\Delta_{b} \leq \beta \leq \Delta_{a}$ & $b e^{\preccurlyeq}(A, B)=T_{w}\left(\lambda_{1}, \delta_{0}, L_{\left(0, \Delta_{a}\right)}^{\preccurlyeq}\left(a_{1}, \max \left(b_{0}+\alpha, a_{0}\right)\right), L_{(\alpha, \beta)}^{\preccurlyeq}\left(a_{0}, b_{0}\right)\right)$ \\
$0<\Delta_{a} \leq \beta \leq \Delta_{b}$ & $b e^{\preccurlyeq}(A, B)=T_{w}\left(\lambda_{1}, \delta_{0}, L_{\left(0, \Delta_{b}\right)}^{\prec}\left(\min \left(a_{1}-\alpha, b_{1}\right), b_{0}\right), L_{(\alpha, \beta)}^{\preccurlyeq}\left(a_{1}, b_{1}\right)\right)$ \\
$0<\Delta_{a} \leq \Delta_{b} \leq \beta$ & $b e^{\preccurlyeq}(A, B)=T_{w}\left(\lambda_{1}, \delta_{0}, L_{(\alpha, \beta)}^{\preccurlyeq}\left(a_{1}, b_{0}\right)\right)$ \\
$0<\Delta_{b} \leq \Delta_{a} \leq \beta$ & $b e^{\preccurlyeq}(A, B)=T_{w}\left(\lambda_{1}, \delta_{0}, L_{(\alpha, \beta)}^{\preccurlyeq}\left(a_{1}, b_{0}\right)\right)$ \\
$0=\Delta_{a} \leq \beta \leq \Delta_{b}$ & $b e^{\preccurlyeq}(A, B)=T_{w}\left(\lambda_{0}, \delta_{0}, L_{\left(0, \Delta_{b}\right)}^{\preccurlyeq}\left(\min \left(a_{0}-\alpha, b_{1}\right), b_{0}\right), L_{(\alpha, \beta)}^{\preccurlyeq}\left(a_{0}, b_{1}\right)\right)$ \\
$0=\Delta_{b} \leq \beta \leq \Delta_{a}$ & $b e^{\preccurlyeq}(A, B)=T_{w}\left(\lambda_{1}, \delta_{1}, L_{\left(0, \Delta_{a}\right)}^{\preccurlyeq}\left(a_{1}, \max \left(b_{1}+\alpha, a_{0}\right)\right), L_{(\alpha, \beta)}^{\preccurlyeq}\left(a_{0}, b_{1}\right)\right)$ \\
$0=\Delta_{a}<\Delta_{b} \leq \beta$ & $b e^{\preccurlyeq}(A, B)=T_{w}\left(\lambda_{0}, \delta_{0}, L_{(\alpha, \beta)}^{\preccurlyeq}\left(a_{0}, b_{0}\right)\right)$ \\
$0=\Delta_{b}<\Delta_{a} \leq \beta$ & $b e^{\preccurlyeq}(A, B)=T_{w}\left(\lambda_{1}, \delta_{1}, L_{(\alpha, \beta)}^{\preccurlyeq}\left(a_{1}, b_{1}\right)\right)$ \\
$0=\Delta_{a}=\Delta_{b} \leq \beta$ & $b e^{\preccurlyeq}(A, B)=T_{w}\left(\lambda_{0}, \delta_{1}, L_{(\alpha, \beta)}^{\preccurlyeq}\left(a_{0}, b_{1}\right)\right)$ \\
\hline
\end{tabular}

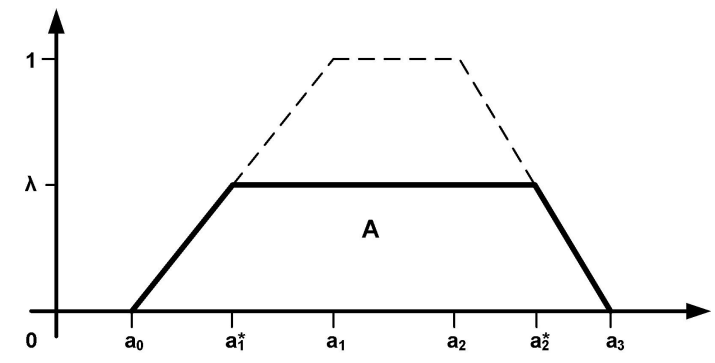

Fig. 5. The fuzzy set $A=\left[a_{0}, a_{1}, a_{2}, a_{3} ; \lambda\right]$

elegant characterization can be obtained for this special class of piecewise linear fuzzy intervals. In the following, we will write $A=\left[a_{0}, a_{1}, a_{2}, a_{3} ; \lambda\right]$ to denote the fuzzy set in $\mathbb{R}$ that is defined for all $x$ in $\mathbb{R}$ as

$$
A(x)= \begin{cases}\frac{x-a_{0}}{a_{1}-a_{0}} & \text { if } a_{0}<x<a_{1}^{*} \\ \lambda & \text { if } a_{1}^{*} \leq x \leq a_{2}^{*} \\ \frac{a_{3}-x}{a_{3}-a_{2}} & \text { if } a_{2}^{*}<x<a_{3} \\ 0 & \text { otherwise }\end{cases}
$$

where $a_{1}^{*}=a_{0}+\left(a_{1}-a_{0}\right) \lambda$ and $a_{2}^{*}=a_{3}-\left(a_{3}-a_{2}\right) \lambda$. Furthermore, we impose $a_{0} \leq a_{1}^{*} \leq a_{2}^{*} \leq a_{3}$ and $\lambda \in[0,1]$. The fuzzy set $A$ is illustrated in Figure 5. In proving properties of such trapezoidally shaped fuzzy sets, the following lemma is often very useful.

Lemma 2: Let $A=\left[a_{0}, a_{1}, a_{2}, a_{3} ; \lambda\right]$. For each $x$ in $\mathbb{R}$ it holds that

$$
A(x)=\min \left(\lambda, L_{\left(0, a_{1}-a_{0}\right)}^{\preccurlyeq}\left(a_{1}, x\right), L_{\left(0, a_{3}-a_{2}\right)}^{\preccurlyeq}\left(x, a_{2}\right)\right)
$$

In the remainder of this paper we will sometimes write $L_{(\alpha, \beta ; \lambda)}^{\ll}(x, y)$ as a shorthand for $\min \left(\lambda, L_{(\alpha, \beta)}^{\ll}(x, y)\right)$, and $L_{(\alpha, \beta ; \lambda)}^{\preccurlyeq}(x, y)$ as a shorthand for $\min \left(\lambda, L_{(\alpha, \beta)}^{\preccurlyeq}(x, y)\right)$. To obtain the characterization for trapezoidally shaped fuzzy intervals, we use the following generalization of Proposition 1.

\section{Proposition 5:}

$$
\begin{aligned}
& L_{\left(\alpha_{1}, \beta_{1} ; \lambda_{1}\right)}^{\ll} \circ L_{\left(\alpha_{2}, \beta_{2} ; \lambda_{2}\right)}^{\ll}=L_{\left(\alpha, \max \left(\beta_{1}, \beta_{2}\right) ; T_{w}\left(\lambda_{1}, \lambda_{2}\right)\right)}^{\ll} \\
& L_{\left(\alpha_{1}, \beta_{1} ; \lambda_{1}\right)}^{\ll} \circ L_{\left(\alpha_{2}, \beta_{2} ; \lambda_{2}\right)}^{\preccurlyeq}=L_{\left(\alpha^{\prime}, \max \left(\beta_{1}, \beta_{2}\right) ; T_{w}\left(\lambda_{1}, \lambda_{2}\right)\right)}^{\preccurlyeq} \circ L_{\left(\alpha_{1}, \beta_{1} ; \lambda_{1}\right)}^{\ll} \circ L_{\left(\alpha_{2}, \beta_{2} ; \lambda_{2}\right)}^{\ll}=L_{\left(\alpha^{\prime \prime}, \max \left(\beta_{1}, \beta_{2}\right) ; T_{w}\left(\lambda_{1}, \lambda_{2}\right)\right)}^{\ll} \\
& L_{\left(\alpha_{1}, \beta_{1} ; \lambda_{1}\right)}^{\preccurlyeq} \circ L_{\left(\alpha_{2}, \beta_{2} ; \lambda_{2}\right)}^{\preccurlyeq}=L_{\left(\alpha^{\prime \prime \prime}, \max \left(\beta_{1}, \beta_{2}\right) ; T_{w}\left(\lambda_{1}, \lambda_{2}\right)\right)}^{\ll}
\end{aligned}
$$

where

$$
\begin{aligned}
\alpha= & \alpha_{1}+\alpha_{2}+\beta_{1} \lambda_{1}+\beta_{2} \lambda_{2}-\max \left(\beta_{1}, \beta_{2}\right) T_{w}\left(\lambda_{1}, \lambda_{2}\right) \\
\alpha^{\prime}= & \alpha_{1}+\alpha_{2}+\min \left(\beta_{1}, \beta_{2}\right)-\lambda_{1} \beta_{1}-\lambda_{2} \beta_{2} \\
& +\max \left(\beta_{1}, \beta_{2}\right) T_{w}\left(\lambda_{1}, \lambda_{2}\right) \\
\alpha^{\prime \prime}= & -\alpha_{1}+\alpha_{2}+\beta_{1} \lambda_{1}+\beta_{2} \lambda_{2}-\beta_{1} \\
& -\max \left(\beta_{1}, \beta_{2}\right) T_{w}\left(\lambda_{1}, \lambda_{2}\right) \\
\alpha^{\prime \prime \prime}= & \alpha_{1}-\alpha_{2}+\beta_{1} \lambda_{1}+\beta_{2} \lambda_{2}-\beta_{2} \\
& -\max \left(\beta_{1}, \beta_{2}\right) T_{w}\left(\lambda_{1}, \lambda_{2}\right)
\end{aligned}
$$

Note that for $\lambda_{1}=\lambda_{2}=1$, Proposition 5 is equivalent to Proposition 1. The following proposition provides a characterization for trapezoidally shaped fuzzy intervals.

Proposition 6: Let $A=\left[a_{0}, a_{1}, a_{2}, a_{3} ; \lambda_{1}\right]$ and $B=$ $\left[b_{0}, b_{1}, b_{2}, b_{3} ; \lambda_{2}\right]$. Furthermore, let $s_{a}^{L}=a_{1}-a_{0}, s_{a}^{R}=$ $a_{3}-a_{2}, s_{b}^{L}=b_{1}-b_{0}$ and $s_{b}^{R}=b_{3}-b_{2}$. It holds that

$$
\begin{gathered}
b b^{\ll}(A, B)=\max \left(T_{w}\left(\lambda_{1}, 1-\lambda_{2}\right),\right. \\
\left.\min \left(\lambda_{1}, L_{\left(\alpha_{1}, \max \left(\beta, s_{a}^{L}, s_{b}^{L}\right)\right)}^{\ll}\left(a_{1}, b_{1}\right)\right)\right) \\
b b^{\preccurlyeq}(A, B)=\min \left(I_{w}\left(\lambda_{2}, \lambda_{1}\right),\right. \\
\left.\max \left(1-\lambda_{2}, L_{\left(\alpha_{2}, \max \left(\beta, s_{a}^{L}, s_{b}^{L}\right)\right)}^{\preccurlyeq}\left(a_{1}, b_{1}\right)\right)\right) \\
e e^{\ll}(A, B)=\max \left(T_{w}\left(\lambda_{2}, 1-\lambda_{1}\right),\right. \\
\left.\min \left(\lambda_{2}, L_{\left(\alpha_{3}, \max \left(\beta, s_{a}^{R}, s_{b}^{R}\right)\right)}^{\ll}\left(a_{2}, b_{2}\right)\right)\right) \\
e e^{\preccurlyeq}(A, B)=\min \left(I_{w}\left(\lambda_{1}, \lambda_{2}\right),\right. \\
\left.\quad \max \left(1-\lambda_{1}, L_{\left(\alpha_{4}, \max \left(\beta, s_{a}^{R}, s_{b}^{R}\right)\right)}^{\preccurlyeq}\left(a_{2}, b_{2}\right)\right)\right) \\
e b^{\ll}(A, B)=\max \left(S_{w}\left(1-\lambda_{1}, 1-\lambda_{2}\right),\right. \\
\left.L_{\left(\alpha_{5}, \max \left(\beta, s_{a}^{R}, s_{b}^{L}\right)\right.}^{\ll}\left(a_{2}, b_{1}\right)\right)
\end{gathered}
$$




$$
\begin{aligned}
& e b^{\preccurlyeq}(A, B)=\max \left(S_{w}\left(1-\lambda_{1}, 1-\lambda_{2}\right),\right. \\
&\left.L_{\left(\alpha_{6}, \max \left(\beta, s_{a}^{R}, s_{b}^{L}\right)\right)}^{\preccurlyeq}\left(a_{2}, b_{1}\right)\right) \\
& b e^{\ll}(A, B)=\min \left(T_{w}\left(\lambda_{1}, \lambda_{2}\right), L_{\left(\alpha_{7}, \max \left(\beta, s_{a}^{L}, s_{b}^{R}\right)\right)}^{\ll}\left(a_{1}, b_{2}\right)\right) \\
& b e^{\preccurlyeq}(A, B)=\min \left(T_{w}\left(\lambda_{1}, \lambda_{2}\right), L_{\left(\alpha_{8}, \max \left(\beta, s_{a}^{L}, s_{b}^{R}\right)\right)}^{\preccurlyeq}\left(a_{1}, b_{2}\right)\right)
\end{aligned}
$$

where

$$
\begin{aligned}
\alpha_{1}= & \alpha+\min \left(0, s_{b}^{L}-\beta\right)\left(1-\lambda_{2}\right)+\max \left(\beta, s_{b}^{L}\right)\left(1-\lambda_{1}\right) \\
& -s_{a}^{L}+\lambda_{1} \min \left(\max \left(\beta, s_{b}^{L}\right), s_{a}^{L}\right) \\
\alpha_{2}= & \alpha+\min \left(0, s_{a}^{L}-\beta\right)\left(1-\lambda_{1}\right)+\max \left(\beta, s_{a}^{L}\right)\left(1-\lambda_{2}\right) \\
& -s_{b}^{L}+\lambda_{2} \min \left(\max \left(\beta, s_{a}^{L}\right), s_{b}^{L}\right) \\
\alpha_{3}= & \alpha+\min \left(0, s_{a}^{R}-\beta\right)\left(1-\lambda_{1}\right)+\max \left(\beta, s_{a}^{R}\right)\left(1-\lambda_{2}\right) \\
& -s_{b}^{R}+\lambda_{2} \min \left(\max \left(\beta, s_{a}^{R}\right), s_{b}^{R}\right) \\
\alpha_{4}= & \alpha+\min \left(0, s_{b}^{R}-\beta\right)\left(1-\lambda_{2}\right)+\max \left(\beta, s_{b}^{R}\right)\left(1-\lambda_{1}\right) \\
& -s_{a}^{R}+\lambda_{1} \min \left(\max \left(\beta, s_{b}^{R}\right), s_{a}^{R}\right) \\
\alpha_{5}= & \alpha+\min \left(0, s_{b}^{L}-\beta\right)\left(1-\lambda_{2}\right)+\min \left(\max \left(\beta, s_{b}^{L}\right), s_{a}^{R}\right) \\
& -\lambda_{2} \max \left(\beta, s_{b}^{L}\right)-s_{a}^{R} \lambda_{1} \\
& +\max \left(\beta, s_{b}^{L}, s_{a}^{R}\right) T_{w}\left(\lambda_{1}, \lambda_{2}\right) \\
\alpha_{6}= & \alpha+\min \left(\beta-s_{b}^{L},\left(\beta-s_{b}^{L}\right)\left(1-\lambda_{2}\right)\right)+\max \left(\beta, s_{b}^{L}\right) \lambda_{2} \\
& +s_{a}^{R} \lambda_{1}-s_{a}^{R}-\max \left(\beta, s_{a}^{R}, s_{b}^{L}\right) T_{w}\left(\lambda_{1}, \lambda_{2}\right) \\
\alpha_{7}= & \alpha+\min \left(\beta-s_{b}^{R},\left(\beta-s_{b}^{R}\right)\left(1-\lambda_{2}\right)\right)+s_{a}^{L} \lambda_{1} \\
& +\max \left(\beta, s_{b}^{R}\right) \lambda_{2}-s_{a}^{L}-\max ^{L}\left(\beta, s_{a}^{L}, s_{b}^{R}\right) T_{w}\left(\lambda_{1}, \lambda_{2}\right) \\
\alpha_{8}= & \alpha+\min \left(0, s_{b}^{R}-\beta\right)\left(1-\lambda_{2}\right)+\min \left(\max \left(\beta, s_{b}^{R}\right), s_{a}^{L}\right) \\
& -\lambda_{1} s_{a}^{L}-\lambda_{2} \max \left(\beta, s_{b}^{R}\right) \\
+ & \max \left(\beta, s_{a}^{L}, s_{b}^{R}\right) T_{w}\left(\lambda_{1}, \lambda_{2}\right) \\
& P r o f: \\
&
\end{aligned}
$$

Proof: (sketch) As an example, we sketch how the characterization for $b b^{\ll}(A, B)$ can be proven. We have

$$
b b^{\ll}(A, B)=\sup _{x} T_{w}\left(A(x), \inf _{y} I_{w}\left(B(y), L_{(\alpha, \beta)}^{\ll}(x, y)\right)\right)
$$

Using Lemma 2 we can show that

$$
\begin{aligned}
\sup _{x} T_{w}\left(A(x), \inf _{y} I_{w}\left(B(y), L_{(\alpha, \beta)}^{\ll}(x, y)\right)\right) \\
=\sup _{x} T_{w}\left(L_{\left(0, a_{1}-a_{0} ; \lambda_{1}\right)}^{\preccurlyeq}\left(a_{1}, x\right),\right. \\
\left.\quad \inf _{y} I_{w}\left(L_{\left(0, b_{1}-b_{0} ; \lambda_{2}\right)}^{\preccurlyeq}\left(b_{1}, y\right), L_{(\alpha, \beta)}^{\ll}(x, y)\right)\right)
\end{aligned}
$$

Using the fact that $1-T_{w}(a, b)=I_{w}(a, 1-b)$ and (1) we find from Proposition 5 (using the notations of this Proposition) that

$$
\begin{gathered}
\inf _{y} I_{w}\left(L_{\left(\alpha_{1}, \beta_{1} ; \lambda_{1}\right)}^{\preccurlyeq}(x, y), L_{\left(\alpha_{2}, \beta_{2} ; \lambda_{2}\right)}^{\ll}(z, y)\right) \\
\quad=L_{\left(\alpha^{\prime}, \max \left(\beta_{1}, \beta_{2}\right) ; T_{w}\left(\lambda_{1}, \lambda_{2}\right)\right)}^{\ll}(z, x)
\end{gathered}
$$

Using (12), and once more Proposition 5 completes the proof.

Note that while we have defined fuzzy intervals as normalised fuzzy sets, in this section we also consider trapezoidally fuzzy sets $A$ for which $h g t A=\sup _{x} A(x)=\lambda<1$. This is useful when we consider unions and intersections of trapezoidally shaped fuzzy sets. For example, it is easy to see that

$$
\begin{aligned}
& b e^{\ll}(A \cup B, C \cup D) \\
& =\max \left(b e^{\ll}(A, C), b e^{\ll}(A, D), b e^{\ll}(B, C), b e^{\ll}(B, D)\right)
\end{aligned}
$$

where $A, B, C$, and $D$ are arbitrary fuzzy sets in $\mathbb{R}$. Moreover, sufficient conditions can be derived under which

$$
\begin{aligned}
& b e^{\ll}(A \cap B, C \cap D) \\
& =\min \left(b e^{\ll}(A, C), b e^{\ll}(A, D), b e^{\ll}(B, C), b e^{\ll}(B, D)\right)
\end{aligned}
$$

However, a detailed discussion of this kind of interactions with union and intersection is outside the scope of this paper.

\section{CONCLUSION}

We have shown how the evaluation of the fuzzy temporal interval relations for piecewise linear fuzzy intervals boils down to the evaluation for linear fuzzy intervals. Furthermore, we have provided a characterization for linear fuzzy intervals that is both efficient to evaluate and easy to implement. Finally, we have provided more elegant definitions for the special case of trapezoidally shaped fuzzy sets.

\section{ACKNOWLEDGEMENT}

Steven Schockaert would like to thank the Fund for Scientific Research - Flanders for funding his research.

\section{REFERENCES}

[1] M. Lapata and A. Lascaride, "Inferring sentence-internal temporal relations," Proceedings of NAACL'04, pp. 153-160, 2004.

[2] J.F. Allen, "Maintaining knowledge about temporal intervals," Соттиnications of the ACM, vol. 26, pp. 832-843, 1983.

[3] D. Dubois and H. Prade, "Processing fuzzy temporal knowledge," IEEE Transactions on Systems, Man, and Cybernetics, vol. 19, pp. 729-744, 1989.

[4] S. Badaloni and M. Giacomin, "A fuzzy extension of Allen's interval algebra," Lecture Notes in Artificial Intelligence, vol. 1792, pp. 155$165,2000$.

[5] D. Dubois, A. HadjAli, and H. Prade, "Fuzziness and uncertainty in temporal reasoning," Journal of Universal Computer Science, vol. 9, pp. 1168-1194, 2003.

[6] G. Nagypál and B. Motik, "A fuzzy model for representing uncertain, subjective and vague temporal knowledge in ontologies," Lecture Notes in Computer Science, vol. 2888, pp. 906-923, 2003.

[7] H.J. Ohlbach, "Relations between fuzzy time intervals," Proceedings of the 11th Int. Symp. on Temporal Representation and Reasoning, pp. 44-51, 2004.

[8] D. Dubois and H. Prade, "Ranking fuzzy numbers in the setting of possibility theory," Information Sciences, vol. 30, pp. 183-224, 1983.

[9] S. Schockaert, M. De Cock, and E.E. Kerrre, "Imprecise temporal interval relations," Lecture Notes in Artificial Intelligence, vol. 3849, pp. 108-113, 2006.

[10] S. Schockaert, M. De Cock, and C. Cornelis, "Relatedness of fuzzy sets," Journal of Intelligent and Fuzzy Systems, vol. 6, pp. 297-303, 2005.

[11] S. Schockaert, M. De Cock, and E.E. Kerre, "Fuzzifying Allen's temporal interval relations," in preparation. 